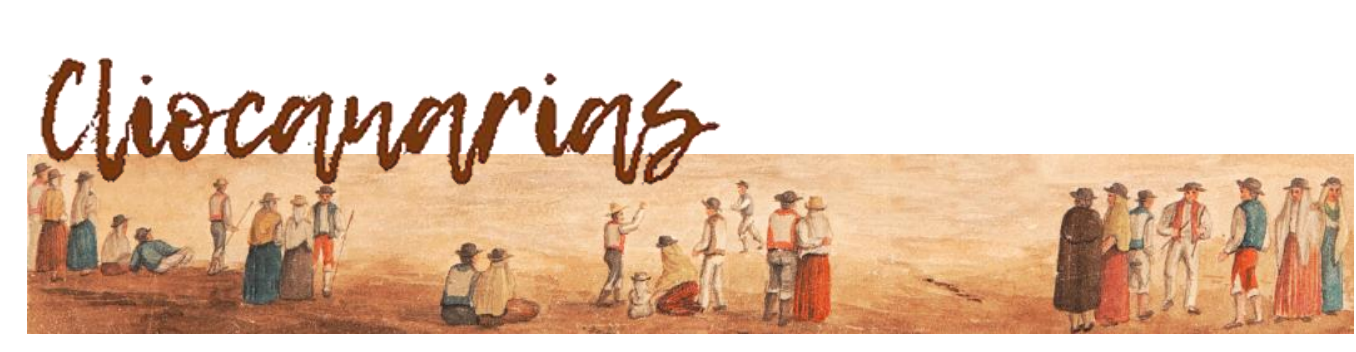

ISSN 2695-4494

https://doi.org/10.53335/cliocanarias.2021.3.20

\title{
HOMO VIDENS: TECNOLOGÍA DIGITAL Y FINALIDADES SOCIOEDUCATI- VAS DE LA ENSEÑANZA DE LA HISTORIA
}

\author{
HOMO VIDENS: DIGITAL TECHNOLOGY AND SOCIO-EDUCATIONAL PURPOSES OF \\ HISTORY EDUCATION
}

Juan Carlos COLOMER RUBIO*

RESUMEN: El presente artículo presenta cómo la implementación de medios tecnológicos digitales ha condicionado la enseñanza de la Ciencias Sociales (Geografia-Historia) especialmente en lo que tiene que ver con la respuesta a las preguntas: ¿Qué enseñar? ¿Para qué? y ¿cómo?. Frente al aceptado lema con tecnología existe innovación educativa, este trabajo pone el énfasis en la importancia de un uso coherente de los medios tecnológicos como complemento a la enseñanza-aprendizaje que necesariamente debe pasar por un conocimiento didáctico y tecnológico del contenido a enseñar.

PALABRAS CLAVE: tecnología educativa, sociedad en red, enseñanza de la historia, medios, contexto educativo.

ABSTRACT: This study presents how the implementation of digital technology has conditioned the teaching of Social Sciences (Geography-History), especially with regard to the answer to the questions: What to teach? What for? and How? Faced with the accepted slogan with technology there is educational innovation, this paper emphasises the importance of a coherent use of technological media as a complement to teaching and learning, which must necessarily involve didactic and technological knowledge of the content to be taught.

KEYWORDS: educational technology, network society, history teaching, media, educational context.

* Profesor de Didáctica de las Ciencias Sociales, Universitat de València. C.e.: juan.colomer@uv.es 


\section{Introducción}

La actual "sociedad red", cuyo desarrollo acelerado debe situarse tras el surgimiento de Internet y su implantación masiva en la década de los 2000, está comportando una serie de cambios fundamentales en la forma de comunicarnos, relacionarnos o aprender. En el seno de esos cambios está el surgimiento de esta nueva sociedad donde el hombre y la mujer se sitúan mediados entre la pantalla, el hiperenlace, la inmediatez de la comunicación o el acceso a multitud de información en constante cambio y transformación ${ }^{1}$. Este homo videns, en palabras de Sartori ${ }^{2}$, no deja de estar influido por una imagen mediática muy poderosa que condiciona la forma de acceder a la realidad. Esto genera claramente problemas en el desarrollo de una ciudadanía consciente y crítica con el mundo en el que vive. Sirva de ejemplo un estudio reciente de la Universidad de Stanford que muestra que un amplio número de jóvenes estadounidenses no saben distinguir una noticia falsa de una verdadera consultada en Internet pese al amplio dominio que dicen mantener de las tecnologias de la información y de la comunicación ${ }^{3}$.

Esta transformación social que afecta a cómo nos comunicamos, nos informamos o generamos nuevo conocimiento deriva de la expansión de los nuevos medios tecnológicos, algo que también ha afectado claramente a lo que se ha venido a denominar la crisis de las humanidades ${ }^{4}$. Esta crisis desarrollada en un nuevo mundo basado en el dato alfanumérico y el peso de la tecnología, que mide continuamente todo lo que hacemos, parece conducir a un retroceso de las ciencias humanas, entre ellas la historia, como herramienta para la crítica social. Por tanto, nos encontramos ante la paradoja de que, en el creciente peso de la información en red, donde la opinión y la información se mezclan, las humanidades tienen problemas de integración, pierden terreno frente a otro tipo de ciencias y su papel llega a ser testimonial en algunos currículos educativos.

Es innegable que estas crisis y transformaciones están afectando claramente a la cuestión: ¿para qué enseñar historia en las aulas? Este nuevo mundo tecnológico ha impactado, como sucede con otras materias, sobre el alumnado y el profesorado. Frente a un alumnado pasivo, con escaso acceso a la información y al que se le suministraban ideas de forma conductista en el pasado, encontramos actualmente un alumnado bombardeado por una gran cantidad de información y subsumido en un mundo tecnológico que le lleva a buscar nuevas respuestas y afirmaciones que se escapan del espacio de la escuela. El acceso a repositorios de información en red construidos de

\footnotetext{
${ }^{1}$ CASTELLS, Manuel: La era de la información: economía, sociedad y cultura, Madrid, Alianza, 1999.

2 SARTORI, Giovanni: Homo videns: la sociedad teledirigida, Madrid, Taurus, 2002.

3 STANFORD HISTORY EDUCATION GROUP): «Evaluating Information: The Cornerstone of Civic Online Reasoning" 29, 2016. Recuperado de: [<https://purl.stanford.edu/fv751yt5934 $>$ ](Fecha de acceso: 31/07/2021).

${ }^{4}$ NUSSBAUM, Martha: Sin fines de lucro: por qué la democracia necesita de las humanidades, Buenos aires, Katz, 2010.
} 
forma colaborativa, como Wikipedia, ha crecido exponencialmente en los últimos años, y en la actualidad es una de las principales fuentes de información de escolares, junto con los libros de texto. El docente, frente a esta nueva realidad, va perdiendo su papel de "transmisor" de contenidos y saberes para pasar a ser dinamizador de las sesiones de aula en un mundo que sabemos cambiante. El escenario derivado de la alerta sanitaria no ha hecho más que acrecentar esta situación.

$\mathrm{El}$ presente texto pretende reflexionar sobra la influencia que ha tenido la inclusión de los medios tecnológicos en el aula de historia, desde la incorporación del cine u otros dispositivos y medios - PLE, plataformas educativas, blogs...- hasta la inserción de metodologías de trabajo basadas, aunque no siempre, en el aprendizaje en red, gamificación, aprendizaje basado en problemas... para ver cómo inexorablemente estas nuevas metodologías y medios están perpetuando unas finalidades socioeducativas sobre las que tradicionalmente se ha ido construyendo la enseñanza y el aprendizaje de la historia en las aulas.

Así, tal y como se expondrá en el texto, la nueva sociedad en red está permitiendo el surgimiento de un nuevo tipo de alumnado inmerso en nuevas pautas de socialización y donde se genera un aprendizaje colaborativo a partir del reconocimiento entre iguales. Esto obliga a un apoyo del docente como mediador y generador de contenidos en red que son modificados y alterados por el estudiante para su aprendizaje. Así, para el caso concreto de la enseñanza de la historia, la tradicional actitud técnica y transmisora de contenidos cerrados, basada en un rol docente de especialista y un papel del alumnado pasivo y memorizador de contenidos empieza a colisionar con la realidad de la nueva normalidad en red que permite la creación de contenidos entre pares de una forma más abierta. Junto con ello, la sociedad que describimos por su propia condición global y transnacional evita el énfasis en contenidos basados en identidades cerradas y homogéneas para apostar por un nuevo tratamiento de la identidad y los problemas del mundo. Pese a todo, la principal dificultad que encontramos es la resistencia a estos cambios, que pueden explicarse por la escasa reflexión sobre medios y metodologías digitales en el aula, lo que ha desarrollado conflictos que pueden verse en las actitudes docentes - muy próximas a la tradición, incluso en el uso de la tecnología- e incluso en las formas de proceder del alumnado, con prácticas alejadas del uso crítico de la tecnología para el desarrollo de una conciencia crítica.

\section{Del libro de texto a TikTok y más allá}

La tecnología ha supuesto un avance significativo en la forma en que los seres humanos nos hemos relacionado con el conocimiento y hemos aprovechado nuestro entorno inmediato. El mismo concepto de "tecnología", tomado del diccionario de la RAE, insiste en ese carácter utilitarista del término además de atemporal: la tecnología ha existido siempre y su desarrollo se ha visto más acelerado y extendido a amplias regiones del mundo desde las últimas revoluciones científicas e industriales. 
Desde el punto de vista educativo, esta realidad tecnológica ha estado presente desde los inicios de la educación reglada como una forma de profundizar en el aprendizaje y obtener conocimiento. La introducción de los libros de texto escolares, en su propia evolución como producto tecnológico, elementos como la pizarra - cuya inserción en la enseñanza se remonta a época romana pero que no fue hasta los inicios de la escuela moderna cuando se popularizó su uso-, o ya recientemente las diapositivas, los proyectores, el ordenador personal, las tabletas electrónicas o las pizarras digitales interactivas (PDI) forman parte de un conjunto tecnológico que está presente y extendido en el mundo educativo. Como vemos, todos estos elementos están encaminados a un mayor aprovechamiento del aprendizaje y del conocimiento, aunque su utilización, en muchas ocasiones, no ha ido orientada hacia ese fin.

Si tomamos por ejemplo el caso de España, en la primera mitad del siglo XIX, mientras se configuraba el sistema educativo liberal, se comenzó a gestar de forma paralela la primera política del libro de texto escolar. Tanto es así que en febrero de 1841 se creaba la primera comisión oficial encargada de examinar estos materiales y, poco después, se publicaba la primera lista de manuales escolares prescritos por el Estado ${ }^{5}$. En este sentido, como prueba clara del binomio libro de texto-disciplina escolar que ha marcado el área de Ciencias Sociales: Geografia e Historia desde los inicios, Raimundo Cuesta advierte que la entrada en vigor del Plan Pidal - el cual, entre otras cosas, decretaba la obligación por parte de los docentes de limitar la elección de los libros de texto al listado elaborado por el Gobierno- se produjo tan solo un mes después de la publicación de los primeros planes de estudio de Historia6.

El papel central del recurso libro de texto desde los inicios de la enseñanza de las Ciencias Sociales es un hecho evidente, el cual se ha visto influido por la orientación educativa de la materia y el tipo de contenidos a enseñar. Así, la priorización de "transmitir" un discurso determinado en el área hizo que en estos materiales se encontrara el mejor soporte sobre el que materializar ese propósito, algo diferente a lo sucedido en otras disciplinas ${ }^{7}$. Pese a su presencia continua en las aulas de Historia desde su constitución como materia escolar (circunstancia que no solo se produjo en el caso español), es evidente que su contenido, recursos y orientación didáctica e historiográfica ha variado sustancialmente por los cambios educativos, políticos, económicos, demográficos... que han tenido lugar. En el caso del discurso, este se ha visto alterado de forma clara con el paso de los años ya que, entre otras cosas, han incidido en él - como comentábamos anteriormente- diferentes finalidades socioeducativas, intereses politicos e identitarios... En fechas más recientes dentro de esta evolución, el relato nacionalizador ha perdido peso a partir de 1970 y se

\footnotetext{
5 DE PUELLES, Manuel: "La política escolar del libro de texto en la España contemporánea", en Avances en supervisión educativa, n. ${ }^{\circ}$ 6, 2007, p. 2.

6 CUESTA, Raimundo: Sociogénesis de una disciplina escolar: la historia, Barcelona, PomaresCorredor, 1997, p.128.

7 STODOLSKY, Susan: La importancia del contenido en la enseñanza. Actividades en las clases de matemáticas y ciencias sociales, Barcelona, Paidós, 1991, pp. 132-135.
} 
han introducido aspectos de la historia económica y social ${ }^{8}$. Todo ello en el marco de la narrativa maestra que vertebra el discurso de los manuales mayoritarios, la cual, en las últimas décadas ha consistido en una metanarrativa de la modernidad que parte de los origenes en la España ilustrada hasta llegar a su integración en la Unión Europea y en el mundo globalizado.

En la actualidad encontramos que los manuales incluyen todo tipo de actividades, recursos o fuentes históricas y muchas de ellas están relacionadas con componentes digitales como webs, portales educativos de la propia empresa editorial o contenedores de información; e incluso se da el caso de libros que adjuntan una URL a entradas concretas de Wikipedia ${ }^{9}$. Además, otra vertiente por considerar es la de los libros de texto digitales, unos materiales que han irrumpido en España de forma desigual según el territorio o tipo de centro y que han terminado por confirmar, en muchos casos, la sospecha de que los libros de texto digitales trasvasan los mismos contenidos, métodos de aprendizaje, tareas, sistemas de evaluación que los libros en papel. De forma que desarrollan todo lo anterior en lugar de aprovechar el cambio de soporte y todas las ventajas de la tecnología digital para incidir en un cambio metodológico que altere los roles tradicionales del docente y del discente, así como la jerarquía subyacente en esos roles sobre la "transmisión" del conocimiento escolar ${ }^{10}$.

En paralelo a la anterior, con la invención de los dispositivos de proyección cinematográfica y su desarrollo, o la aparición de la televisión en los años 30 y 40 del siglo $\mathrm{xx}$, los libros de texto fueron progresivamente complementados por la utilización de imágenes proyectadas. Esto llevó, por ejemplo, a la utilización del cine como herramienta en las clases de historia o geografia, especialmente cuando comenzó a verse no como un sistema técnico o una explosión de efectos deslumbrantes sino una representación del mundo con contenido histórico y con un discurso determinado que podía ser trabajado desde el aula ${ }^{11}$. La inclusión de estos elementos supuso una auténtica revolución en la docencia, pues facilitaba poner en movimiento muchas de las ideas proyectadas de forma fija en otros soportes tecnológicos, además de insistir en una nueva forma de ver el mundo y la realidad social.

Así, la incorporación del cine como herramienta didáctica ya planteó una serie de problemáticas asociadas que fueron estudiadas específicamente en

\footnotetext{
8 Puede encontrarse un análisis completo de estos aspectos, junto con muchos otros, en el capítulo 3 de VALLS, Rafael: Historiografía escolar española: siglos XIX-XXI, Madrid, UNED, 2007.

9 Un ejemplo de actividades que remiten a Wikipedia puede verse en el manual de la Editorial Edelvives, PROYECTO PIXÉPOLIS: Conocimiento del Medio, Tercer trimestre, 6 Primaria. Madrid, Edelvives, 2013, que en una de las tareas demanda al alumnado lo siguiente: "Averigua qué es la Ley Sálica en Wikipedia y explica de qué manera influyó en la guerra entre liberales y carlistas" (p. 183).

10 PEIRATS, José, Isabel María GALLARDO, Ángel SAN MARTíN y María José WALIÑO: "Análisis de la industria editorial y protocolo para la selección del libro de texto en formato digital", en Profesorado. Revista de currículum y formación del profesorado, n. ${ }^{\circ} 20,2016,2016$, pp. 7589.

${ }^{11}$ BELLIDO, Adolfo: «El aprendizaje del cine», en Comunicar, n. ${ }^{\circ} 11,1998$, p.15.
} 
trabajos de Ferro, Sorlin, Caparros Lera, Gispert, Seixas, entre otras personas ${ }^{12}$. Estos estudios ya sugerian de qué modo el cine se habia incorporado como un elemento muy interesante y se constituía en un potente instrumento para el trabajo de determinados contenidos sociales, junto con una poderosa fuente que podia ser analizada con herramientas críticas y una metodología clara. El docente, en muchos casos, utilizó esta innovación desde varios enfoques diferentes: de forma accesoria para cubrir alguna idea o concepto no trabajado desde otra perspectiva, o usado como material o fuente que podía ser analizada con los útiles propios del trabajo de historiador. El cine y otros recursos audiovisuales tuvieron una incidencia en el papel, cada vez mayor, de la multimedia expositiva en las clases de historia y de geografia, recurriendo a una combinación de diferentes canales de comunicación (visual, auditivo...) y a una diversidad de tipologias de información (textos, imágenes, animaciones...) $)^{13}$. Por tanto, esta multimedia incluiria desde sistemas de enseñanza en linea interactiva hasta proyecciones audiovisuales integradas en la sesión educativa en el aula.

El segundo gran hito, tras la inclusión del cine y de las dinámicas multimedia en Ciencias Sociales, debe buscarse en la inserción del ordenador, especialmente, como ha señalado Rivero para el caso español, a mediados de los años ochenta del siglo XX. A los primeros modelos como el Commodore 64, el Spectrum o los primeros Macintosh, más sencillos en su manejo que los PC con sistema operativo DOS, le siguió la progresiva implantación del sistema operativo Windows, inspirado en el de Macintosh, y que se convirtió en el sistema predominante en el mercado hasta la actualidad pese a la existencia de sistemas operativos de software libre como Linux en muchos centros escolares. Esta inserción de ordenadores en las aulas se vio complementada por varios programas ministeriales como el proyecto Atenea o Mercurio en la década de los ochenta, la fusión de ambos con el Programa Nacional de Tecnologías de la Información y Comunicación, o posteriormente el programa Escuela 2.0 en $2009^{14}$. Además, se impulsaron varios centros de formación e investigación educativa, como el propio INTEF en 2012, al que le han seguido otras iniciativas de formación reciente como los MOOC (Massive Open Online Course, o cursos en línea masivos abiertos) del propio instituto.

\footnotetext{
12 FERRO, Marc: Cine e historia, Barcelona, Gustavo Gilli, 1980; SORLIN, Pierre: Sociología del cine: la apertura para la historia de mañana, México, Fondo de Cultura Económica, 1992; CAPARRóS LERA, José Maria: Guía del espectador de cine, Madrid, Alianza, 2007; GISPERT, Esther: Cine, ficción y educación, Barcelona, Laertes, 2009; SEIXAS, Peter: "Popular film and young people's understanding of the history of native-white relations", en The History Teacher, n. ${ }^{\circ}$ 26, 1993, pp. 351-370; SEIXAS, Peter: "Confronting the moral frames of popular film: Young people respond to historical revisionism", en American Journal of Education, n. ${ }^{\circ}$ 102, 1994, pp. 261-285.

${ }^{13}$ RIVERO, Pilar: La eficiencia didáctica en el aprendizaje de la Historia en $1 .^{\circ}$ de ESO mediante Nuevas Tecnologías básicas. Tesis doctoral (inédita), Barcelona, Universitat de Barcelona, 2009, p. 35.

${ }^{14}$ Una reflexión amplia sobre estos programas de inserción de los ordenadores en las aulas puede leerse en AREA, Manuel: "Una breve historia de las políticas de incorporación de las tecnologías digitales al sistema escolar en España", en Quaderns Digitals, n. ${ }^{\circ}$ 51, 2008. [<https://goo.gl/OlJF5R>] (Fecha de acceso: 31/07/2021).
} 
Gracias a estos planes de inserción, el peso de los dispositivos digitales en las aulas fue en aumento en paralelo a su inclusión en muchos domicilios. En los centros escolares fueron ganando peso, entre otras razones, por la presumible autonomía que ofrecían al alumno en la realización de tareas y a las posibilidades que daba al docente para la "transmisión" de determinados contenidos utilizando las ventajas de estos dispositivos. Tal es su importancia que, aún hoy, observamos una tendencia ascendente en muchos centros escolares en incorporar este tipo de recursos en forma de tabletas y otros dispositivos digitales, aspecto que la pandemia derivada de la COVID-19 ha incrementado, en una estrategia de progresiva o total desaparición de los libros de texto en papel.

La implementación de Internet en la escuela, inicialmente en las denominadas aulas de informática, por medio, en muchos casos, de tecnología sin cables o wifi, llevó al desarrollo de actividades concretas con los ordenadores como las propias WebQuest, entendidas como tareas atractivas desarrolladas por el docente y que el alumnado debía realizar administrando su propio tiempo y utilizando las propias herramientas que le aportaba la red de redes. Este tipo de actividades implementadas de forma extensa en primera década de este siglo para el aprendizaje o tratamiento de determinados conceptos históricos o geográficos han sido abordadas en varias investigaciones y fueron los primeros recursos incorporados de forma masiva tras la inserción de Internet en los centros escolares ${ }^{15}$. Estas actividades mejoraron y se multiplicaron tras el desarrollo de la web 2.0 que planteaba una mayor capacidad de interacción con el usuario, dándole al alumnado la posibilidad — si se estaban utilizando, por ejemplo, espacios wiki o blogs - de generar sus propios materiales y discursos concretos sobre los más variados contenidos. Entre las últimas novedades incorporadas en las clases de Ciencias Sociales se encuentra el uso de los videojuegos o vídeos en streaming para el trabajo de determinados contenidos, la utilización de redes sociales como Tiktok o Instagram en conexión con métodos o recursos supuestamente novedosos como la gamificación o el Flipped Classroom ${ }^{16}$. Todo lo expuesto se ha complementado con la inserción de nuevos materiales, como las propias enciclopedias en red, o repositorios de

\footnotetext{
15 MILSON, Andrew, J.: "The internet and inquiry learning: Integrating medium and method in a sixth grade social studies classroom", en Theory and Research in Social Education, n. ${ }^{\circ} 30$, 2002, pp. 330-353; LEE, John K., y Brendan CALANDRA: "Can embedded annotations help high school students perform problem solving tasks using a web-based historical document?", en Journal of Research on Technology in Education, n. ${ }^{\circ}$ 37, 2004, pp. 65-84; CÓZAR, Ramón: "La WebQuest en la enseñanza/aprendizaje de las Ciencias Sociales", en CÓZAR, Ramón, y Maria DEL VALLE DE MOYA (coord.): Las TIC en el aula desde un enfoque multidisciplinar: aplicaciones practices, Barcelona, Octaedro, 2013, pp. 45-62.

16 SÁNCHEZ, Maria: "Redefinir la historia que se enseña a la luz de las TIC: un análisis sobre nuevas maneras de aprender Roma", en VERA-MUÑOZ, Isabel, y David PÉREZ I PÉREZ, Formación de la ciudadania: las TICs y los nuevos problemas, Alicante, Universidad de Ali-cante, 2004; CUENCA, José Maria, y Myriam MARTÍN: "La resolución de problemas en la en-señanza de las ciencias sociales a través de videojuegos", en Íber: Didáctica de las Ciencias Sociales, Geografía e Historia, n. ${ }^{\circ}$ 63, 2010, pp. 32-42; RIVERO, Pilar: "Procesos de gamificación en el aula de ciencias sociales", en Íber: Didáctica de las Ciencias Sociales, Geografía e Historia, n. ${ }^{\circ}$ 86, 2017, pp. 4-6.
} 
documentación, audios o vídeo que han supuesto una ampliación de contenidos al libro de texto al que llegan a apoyar pero nunca sustituyen por completo $^{17}$.

Junto con estos recursos, la inserción de Internet ha llevado a la generación de plataformas en red que, vinculadas nuevamente a dispositivos (como el caso del iPad o la pizarra digital), o complementando tecnologias emergentes como la realidad aumentada o la virtualización, suponen las innovaciones más recientes en nuestra área. Estas plataformas y métodos han insistido en la perspectiva de inmediatez que la tecnología aporta para el trabajo con recursos digitales, pero mayoritariamente lo han hecho sin la consiguiente reflexión metodológica y epistemológica necesaria para el desarrollo de una ciudadanía crítica.

La introducción de los sistemas informáticos y, especialmente tras la expansión de Internet, la inclusión de la tecnología en las aulas ha tenido una evolución aún mayor, pues ha supuesto el menoscabo progresivo de los espacios físicos para apostar por una educación interconectada basada en los entornos interactivos de aprendizaje o personales (PLE) que se han ido incorporando progresivamente a los espacios, a los materiales y al currículo oficial. Estos dispositivos tecnológicos permiten emplear en el aula infinidad de nuevos recursos que pueden ser alterados y modificados en red. Además, la aparición de contenedores de información que pueden ser manipulados por el propio usuario -Wikipedia, por ejemplo- condiciona la enseñanza tradicional de conocimiento (del docente al discente), para pasar a ser un conocimiento compartido que se genera autónomamente y en red ${ }^{18}$. En paralelo a lo anterior, esto ha alterado el papel del profesorado en muchas disciplinas escolares, en cuanto este no atesora la centralidad del conocimiento como habia ocurrido en épocas pasadas. De ese modo se rescatan pedagogías donde el conocimiento compartido entre los agentes de la comunidad educativa resulta fundamental para la construcción del conocimiento escolar. Así, desde estos entornos se ha planteado vincular metodologias como la de la coasociación (figura 1) con el trabajo asistido de tecnología en las aulas, una dinámica basada en que el estudiante es socio en su proceso de aprendizaje sirviéndose de habilidades distintas de las habituales, pero que resultan compatibles y enriquecedoras $^{19}$.

\footnotetext{
17 Según datos del informe elaborado por la ANELE (Asociación Nacional de Editores de Libros y material de Enseñanza) en 2020: Año tras año queda patente que la oferta editorial de libros de texto digitales, en sus diversos tipos, se incrementa de manera exponencial, mientras que las ventas o se estancan o crecen muy ligeramente y representa una minima parte de la facturación del sector. $\mathrm{El}$ informe completo puede consultarse en la propia página web de ANELE: [<https://anele.org/wp-content/uploads/2020/09/200911TXT-ANELE-La-edicion-educativa-20-21.pdf>] (Fecha de acceso: 31/07/2021).

18 Para una interesante reflexión sobre el papel de Wikipedia como generador del conocimiento que se crea y se comparte en la red, véase: PONS, Anaclet: El desorden digital. Guía para historiadores y humanistas, Madrid, Siglo XXI, 2013, pp. 129-161.

19 PRENSKY, Marc: Enseñar a nativos digitales, Madrid, Ediciones SM, 2011.
} 


\section{En la forma está la respuesta... utilizar la tecnologia para cambiar la práctica}

El peso y la centralidad de los dispositivos, desde el libro, el proyector o el ordenador ha ido obviando las ventajas de las adecuadas metodologias para el trabajo de las Ciencias Sociales en el aula como la presentada en la figura 1 .

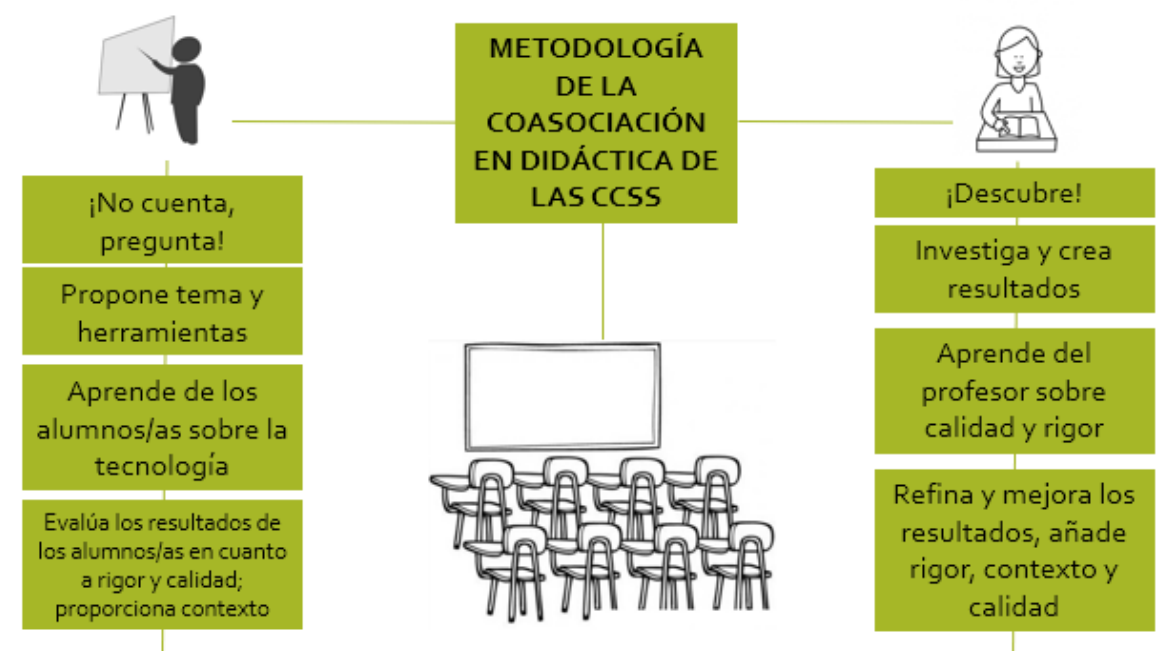

Figura 1. Metodología de la coasociación en didáctica de las Ciencias Sociales (Fuente: elaboración propia a partir de PRENSKY, Marc: Enseñar a nativos digitales, Madrid, Ediciones SM, 2011, 2011).

Con la progresiva evolución técnica de estos medios y un intento de formación del profesorado que se ha visto deficiente y poco estructurado, se ha considerado necesaria una reflexión que vaya encaminada hacia qué metodología es más conveniente para el trabajo con el alumnado, de forma que conecte con las propias finalidades socioeducativas emancipadoras que propugna el modelo crítico defendido en la disciplina desde hace algunos años.

Si tomamos el caso de las Ciencias Sociales, tal y como han señalado Arancibia y Badia, vemos que un determinado uso de las TIC está ampliamente relacionado con prácticas pedagógicas determinadas que ya venían de modelos anteriores al surgimiento de la tecnología digital ${ }^{20}$. Por un lado, estos autores señalan teorías del procesamiento de la información que se aproximan a una concepción transmisiva del contenido disciplinar mediante un uso del ordenador o de dispositivos portátiles de una manera conductista. Esta metodología tiene el trasfondo de una enseñanza que se dedica a entregar información y a mostrar la realidad como elementos externos al sujeto, persiguiendo un aprendizaje que debe ser fiel reproducción (una copia) de esa realidad externa. Este modelo es el que impera claramente en la utilización de determi-

20 ARANCIBIA, Marcelo y Antoni BADIA: "Caracterización y valoración de los usos educativos de las TIC en 10 secuencias didácticas de historia en enseñanza secundaria", en Estudios Pedagógicos, número especial, 2013, pp. 7-24. 
nadas técnicas en el aula de Ciencias Sociales, como puede quedar de manifiesto en el abuso de las presentaciones digitales o de vídeos sin la consiguiente reflexión didáctica o epistemológica.

Por otro lado, Arancibia y Badia plantean otro enfoque como es la utilización de la tecnología proveniente de las herramientas cognitivas asociadas al constructivismo individual, pues asume un tipo de enseñanza en que el estudiante decide itinerarios, actividades y metas, lo que supone un uso de la tecnología como apoyo al desarrollo cognitivo individual, asociado a una concepción sobre aprender y enseñar que sitúa el foco en los intereses individuales de los estudiantes mediante plataformas o aplicaciones determinadas. Esta forma de trabajo, menos condicionada que la anterior, señala prácticas que no dependen tanto de la aplicación o de los contenidos asociados a la misma, sino más bien la orientación docente en cada una de las actividades sugeridas.

Por último, se ha insistido en otras dimensiones como la tendencia a utilizar entornos abiertos de aprendizaje, los cuales se ubican también dentro del paradigma constructivista pero con un enfoque social, ya que apuestan por la configuración de una enseñanza que favorezca la interacción educativa así como la construcción colectiva del conocimiento y, por tanto, un aprendizaje participativo y colaborativo en la línea de pedagogias como la de la coasociación de Prensky (figura 1), ya citada anteriormente. Así, la utilización de aplicaciones y recursos pensados o no con fines educativos, unido a unos planteamientos docentes innovadores donde se construya conocimiento de forma colaborativa, puede resultar una de las claves adecuadas para una correcta inserción de la tecnología en las aulas.

Como vemos, este proceso de reflexión metodológica acentúa cada vez más las competencias de los docentes y su papel como mediadores entre la tecnología disponible y el aprendizaje del alumnado. Por tanto, una metodología efectiva siempre vendrá de la mano de un amplio conocimiento, no solo tecnológico, sino de los contenidos pedagógicos y disciplinares a él asociados.

Para ello, ya en los años ochenta del siglo xx, el grupo de investigación Knowledge Growth in a Profession: development of knowledge in teaching, dirigido por Shulman, de la Universidad de Standford, consideró conveniente insistir en el papel del docente como articulador de conocimiento dentro del aula. Así se destacaron aspectos como el conocimiento base del profesor, que incluye tanto la dimensión conceptual (saber qué) y pedagógica como la dimensión procedimental (saber hacer), que contiene, al mismo tiempo, las justificaciones para que se actúe de determinada forma (por qué) ${ }^{21}$.

Sobre esta propuesta se consolidó desde el ámbito norteamericano el modelo TPACK (Technological Pedagogical Content Knowledge), planteado por Mishra y Koehler en 2006, que se insertó en las aulas mediante el diagnóstico y la combinación de contenidos, pedagogía y tecnología ${ }^{22}$. Este modelo parte del trabajo de Shulman para señalar que la tecnología como única respuesta no

${ }^{21}$ SHULMAN, Lee S.: "Those who understand: knowledge growth in teaching", en Educational Researcher, n. ${ }^{\circ} 15,1986$, pp. 4-14.

22 MISHRA, Punya, y Matthew KOEHLER: "Technological pedagogical content knowledge: a framework for teacher knowledge", en Teachers College Record, n. ${ }^{\circ}$ 108, 2006, pp. 1017-1054. 
puede suponer una mejora en el aprendizaje por sí misma, sino que necesita de complementos pedagógicos y epistémicos. Así, el TPACK da respuesta al creciente interés de integrar la tecnologia en el servicio de los procesos formativos, generando una serie de interrelaciones e interacciones entre las tres fuentes primarias de conocimiento: disciplinar, pedagógico y tecnológico.

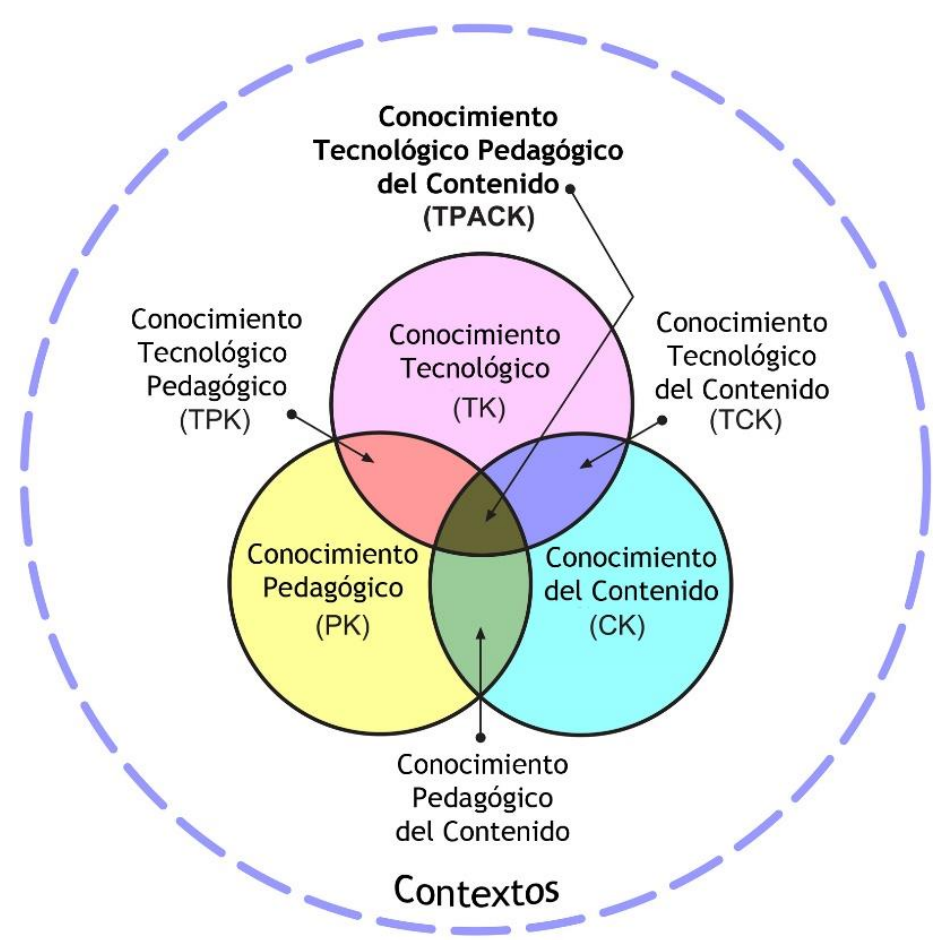

Figura 2: Esquema del modelo TPACK

(Fuente: http://www.tpack.org/)

Así, siguiendo este modelo (figura 2), una correcta inserción de la tecnología educativa en las aulas, también de Ciencias Sociales, pasaría por el dominio de tres tipos de conocimiento: tecnológicos (TK), pedagógicos (PK), y de contenidos o disciplinar (CK). Ahora bien, lo verdaderamente significativo que propone el modelo no es la formación independiente en estos tres tipos de conocimientos (CK-PK-TK), sino que también deben percibirse en relación e interacción constante: PCK: conocimiento pedagógico del contenido; TCK: conocimiento de la utilización de las tecnologias; TPK: conocimiento pedagógico tecnológico en un contexto cambiante. En suma, surgiría el TPACK: conocimiento tecnológico, pedagógico y de contenido como simbiosis de todos estos conocimientos ${ }^{23}$.

Asimismo, tal y como ha señalado Gómez, este modelo insiste en que para llevar a cabo una correcta enseñanza con tecnología es necesaria la adquisición y comprensión de tales interacciones entre los diferentes elementos. Así pues, la inclusión de la tecnología en las aulas de Ciencias Sociales necesitaría

23 Este modelo ha generado buenos instrumentos para evaluar cada uno de los conocimientos en docentes de cualquier ámbito educativo: CABERO, Julio, Verónica MARÍN y Carlos CASTAÑO: "Validación de la Aplicación del modelo TPACK para la formación del profesorado en TIC», en @Tic. Revista D’Innovació Educativa, n. ${ }^{\circ} 22,2015$, pp. 13-22. 
una adecuada formación respecto de los dispositivos digitales no sólo de tipo instrumental sino, muy especialmente, de los contenidos de la disciplina que se va a trabajar y de las metodologías-pedagogias concretas que se quieren implementar para la consecución de los objetivos didácticos pretendidos ${ }^{24}$.

Todo ello se complementa con el papel del alumnado, donde su rol pasivo y receptor de conocimiento se modifica para ser el constructor de su propio aprendizaje. Aquí la amplia mayoría de recursos en red, algunos de software libre que permiten alterar su código fuente para acercarlo a las necesidades de los usuarios, facilitan o empoderan al discente en la consecución de sus propios objetivos de aprendizaje. Esto, como han sugerido Rivero y Mur para el caso de las Ciencias Sociales, hace que el alumnado se enseñe entre sí y aprenda de forma autónoma, realizando tareas mediadas por la tecnologia como: buscar información, plantear hipótesis, encontrar respuestas y crear presentaciones, las cuales pueden ser, por ejemplo, valoradas posteriormente por el colectivo docente y la clase según su corrección, contexto, rigor y calidad, de manera que si se plantea una actividad adecuadamente diseñada se cubre el curriculo porque las preguntas que responden los alumnos son las que necesitan conocer ${ }^{25}$.

\section{A modo de conclusión}

Tecnología es un todo y su evolución ha estado en la base de cualquier tradición educativa. También, como hemos ido señalando en estas páginas, a lo largo de la historia "enseñada", esta misma tecnología ha estado presente de una forma u otra en el aprovechamiento del conocimiento de generaciones de docentes y escolares. Desde la pizarra, pasando por el libro o los nuevos sistemas electrónicos, todo ha sido y es técnica de aprovechamiento y extracción de los conocimientos disponibles para la "transmisión" de habilidades básicas de conocimiento. Pese a todo, como hemos destacado, su grado de importancia ha sido muy desigual. Al peso determinante del libro de texto le ha seguido una serie de complementos tecnológicos - como el cine o los dispositivos informáticos- que, pese a hacer marcado hitos fundamentales en el desarrollo de la disciplina, no han conseguido desplazar el omnimodo papel del manual ni las prácticas y rutinas escolares o tradicionales a él asociadas. La incorporación progresiva de distintos elementos digitales ha derivado actualmente en nuevas aplicaciones y entornos que alteran (como ya ha sucedido en otras ocasiones) las propias bases sobre las que hemos construido la enseñanza de la Historia y la Geografia, entre otras materias.

${ }^{24}$ GÓMEZ, Isabel Maria: "La adquisición del conocimiento base del docente en Ciencias Sociales a través del modelo de enseñanza y aprendizaje TPACK en la formación inicial del profesorado con tecnologia", en Ramón MARTÍNEZ, Roberto GARCÍA-MORIS y Carmen Rosa GARCÍA (eds.): Investigación en Didáctica de las Ciencias Sociales. Retos, Preguntas y Líneas de Investigación. Córdoba, Universidad de Córdoba, 2017, pp. 151-160

${ }^{25}$ RIVERO, Pilar, y Lorenzo MUR: "Aprender ciencias sociales en la web 2.0", en Íber: Didáctica de las Ciencias Sociales, Geografía e Historia, n. ${ }^{\circ}$ 80, 2015, p. 36. Los autores se apoyan en las reflexiones de Prensky ya citadas. 
Esta modificación se ha generado debido a la inclusión de prácticas que han ido enfatizando los recursos tecnológicos, a los que se ha dado una importancia fundamental, pues permitian un supuesto aprendizaje autónomo del discente y un papel secundario del profesorado. Esto ha derivado, en muchas ocasiones, en una utilización de la tecnología como premio al alumnado desmotivado, accesorio en las dinámicas de aula o mero instrumento al servicio del docente que, con formación escasa en "nuevas tecnologías", se movía como podía en el "marasmo digital". Se ha confundido, en definitiva, la innovación con la utilización de la tecnología en el aula y esto ha deparado para no pocos resultados negativos.

Hoy muchos de estos problemas se han convertido en desafios crecientes dentro de nuestra disciplina de conocimiento. Así, teniendo en cuenta los últimos informes Horizon elaborados por New Media Consortium (NMC) y Consortium for School Networking (CoSN), que tienen en cuenta las tendencias y desafíos en novedades tecnológicas de los próximos años en el mundo educativo, estimamos conveniente apuntar cómo, en un futuro inmediato, la inmersión tecnológica va a necesitar de un replanteamiento del rol del docente y, lo que es más importante, una formación más personalizada —como la sugerida con el modelo TPACK - que permita igualar las condiciones de acceso y de uso de los discentes a la tecnología. A ello debemos añadir otros desafíos como las consecuencias de la pandemia en el mundo de la educación digital, la convivencia de diferentes dispositivos y recursos en las aulas, en muchos casos poco dotadas o actualizadas a las aplicaciones que se pretende manejar, o la escasa formación del profesorado que, a veces, se suple con modas pedagógicas que no contienen una reflexión crítica. El alumnado, expuesto continuamente a un bombardeo de la tecnología digital, puede llegar a plantear contradicciones en sus prácticas diarias de relación con la misma escuela que, o no sabe adaptarse a los cambios, o se cierra a ciertas innovaciones que necesariamente vienen impuestas por el nuevo contexto en que nos movemos.

Ciertamente, para un uso crítico de la tecnología, especialmente en las clases de Geografia e Historia, debemos avanzar en lo sugerido por Kozma cuando afirmaba que la educación escolar en este nuevo escenario debe plantearse una modificación de sus finalidades dirigiéndolas más allá de sus objetivos de alfabetización básica en contenidos hacia aspectos relacionados con la comprensión profunda del conocimiento disponible y a la creación de nuevo conocimiento, la redefinición y resolución de problemas complejos o el uso de las tecnologias digitales como instrumento de aprendizaje ${ }^{26}$. Aquí, como se viene planteando desde los fundamentos de una pedagogía crítica y reflexiva, nuestro campo de conocimiento puede aportar una reflexión fundamental con tecnología en esa nueva orientación que necesariamente condicionará la práctica de enseñar y aprender en el siglo XXI.

${ }^{26}$ KOZMA, Robert B. (2012): Las TIC y la transformación de la educación en la economía del conocimiento. En: [<http://www.debats.cat/es/debates/las-tic-y-la-transformacion-de-laeducacion-en-la-economia-del-conocimiento>] (Fecha de acceso: 31/07/2021). 